\title{
Aplicación de análisis discriminante para evaluar el mejoramiento de los indicadores financieros en las empresas del sector alimento de Barranquilla-Colombia
}

\author{
Application of discriminant analysis to evaluate the improvement of financial \\ indicators in the food sector companies Barranquilla-Colombia
}

\author{
Tomás Fontalvo Herrera ${ }^{1} \quad$ Efraín De la Hoz Granadillo ${ }^{1} \quad$ Juan Carlos Vergara ${ }^{1}$
}

Recibido 26 de marzo de 2012, aceptado 24 de agosto de 2012

Received: March 26, 2012 Accepted: August 24, 2012

\begin{abstract}
RESUMEN
En este artículo de investigación se presentan los resultados obtenidos durante el desarrollo del proyecto Aplicación del análisis discriminante para evaluar el mejoramiento de los indicadores financieros en las empresas del sector alimento de Barranquilla. En este se realiza inicialmente una descripción detallada de la evaluación de los sistemas organizacionales, los indicadores de liquidez, indicadores de actividad, indicadores de rentabilidad e indicadores de endeudamiento, los criterios para la aplicación del análisis discriminante en la evaluación del mejoramiento de los indicadores de liquidez, actividad, rentabilidad y endeudamiento en las empresas del sector alimento de Barranquilla y la utilización de la técnica análisis discriminante para diferenciar dos periodos establecidos, 2004 y 2009. Lo anterior a través de una perspectiva cualitativa, descriptiva, propositiva y cuantitativa. De la función discriminante obtenida y de los estadísticos analizados se puede concluir que los indicadores financieros Rentabilidad Operativa del Activo (RO), Rotación de Activo (RA), Nivel de Endeudamiento (NE) del sector presentan diferencias significativas de un periodo a otro. Aunque solo la media del indicador financiero Apalancamiento a Largo Plazo (ALP) mejora de un periodo a otro. Finalmente se pudo estructurar una metodología de análisis robusta que evalúa la evolución de los resultados financieros obtenidos en las organizaciones empresariales siendo de gran utilidad y valor como referencia científica en el análisis de indicadores financieros para la toma de decisiones gerenciales.
\end{abstract}

Palabras clave: Análisis discriminante, liquidez, actividad, rentabilidad, endeudamiento.

\section{ABSTRACT}

In this research paper are presented the results obtained during the project Application of discriminant analysis to evaluate the improvement of financial indicators in the food sector companies in Barranquilla, this is initially a detailed description evaluation of the organizational systems, liquidity ratios, activity indicators, profitability indicators and debt indicators, the criteria for the application of discriminant analysis in evaluating the improvement of liquidity ratios, activity, profitability and debt in the food sector companies Barranquilla and the use of discriminant analysis technique to distinguish two periods established 2004 and 2009. This perspective through qualitative and quantitative descriptive propositional. Discriminant function obtained and analyzed the statistics we can conclude that the financial indicators Operating Assets Return (AS), Asset Turnover (AT), Level of Indebtedness (LI) sector, significant differences from one period to another. Although only financial indicator the average long-term leverage $(A L L)$ improved from one period to another. Finally, we could structure a robust analysis methodology that evaluates the evolution of financial results in business organizations is of great use and value as a scientific reference on the analysis of financial indicators for management decision making.

Keywords: Discriminant analysis, liquidity, activity, profitability, indebtedness.

1 Universidad de Cartagena. Av. El Consulado, Calle 30 No $^{\circ}$ 48-152. Cartagena 1382. Cartagena de Indias, Colombia. E-mail: tfontalvoh@unicartagena.edu.co; edelahozg@unicartagena.edu.co; jvergaras@unicartagena.edu.co 


\section{INTRODUCCIÓN}

En este artículo se desarrolló una metodología para evaluar el mejoramiento de los indicadores financieros en las empresas del sector alimento de Barranquilla-Colombia, tomando para este estudio aquellas empresas que presentaron sus estados financieros en la Superintendencia de Sociedades y la Cámara de Comercio de Barranquilla-Colombia en los periodos 2004 y 2009.

Con el fin de realizar una evaluación detallada de las empresas, se utilizó el análisis discriminante tomando razones financieras y definiendo las variables dependientes e independientes que permitieron disminuir la varianza dentro de los grupos y maximizar la varianza entre grupos, para lo cual se utilizaron los indicadores de liquidez, actividad, rentabilidad y endeudamiento, con lo cual evaluó el desempeño de las compañías de tal forma que se pudiera analizar la relación e incidencia en los indicadores financieros seleccionados.

Un estudio de esta naturaleza implica la revisión conceptual y teórica sobre los sistemas organizacionales, los indicadores de liquidez, actividad, rentabilidad y endeudamiento, los criterios para evaluar el mejoramiento de los indicadores financieros, la técnica análisis discriminante, la distancia $\mathrm{D}^{2}$ de Mahalanobis y las pruebas de validez. A partir del marco teórico se estructuró un modelo que sirvió de fundamento en la aplicación del análisis discriminante para evaluar el mejoramiento de los indicadores financieros de las empresas del sector alimento de Barranquilla. Seguidamente se expone la metodología de investigación empírica y se presentan los resultados por medio de la contrastación de hipótesis de las variables asociadas al modelo. Por último, se aportan las conclusiones asociadas a la precisión del modelo y a la evaluación de los indicadores seleccionados de las empresas de Barranquilla.

Es importante señalar que como resultado de la investigación se presenta un método y un conjunto de herramientas que permiten la evaluación de la evolución de los diferentes indicadores financieros en las empresas del sector alimento de Barranquilla. Además se presenta una explicación clara del análisis de las diferentes variables y rubros asociados a los indicadores seleccionados, frente a los resultados alcanzados en los periodos 2004 y 2009.

\section{ANÁLISIS TEÓRICO}

\section{Evaluación de sistemas organizacionales}

La evaluación de organizaciones supone un cuerpo de conocimientos teóricos y metodológicos, así como un conjunto de habilidades aplicadas. Para esta investigación este cuerpo de conocimientos lo constituyen los fundamentos de Análisis Discriminante Multivariado (ADM), que permiten evaluar el mejoramiento de los indicadores de liquidez, actividad, rentabilidad y endeudamiento en las empresas del sector alimento de Barranquilla [1-2].

\section{Indicadores financieros}

Para analizar si en dos periodos distintos se puede evidenciar la evolución de los estados financieros como resultado de la evaluación del mejoramiento de los indicadores de liquidez, actividad, rentabilidad y endeudamiento en el sector analizado, se utilizó la técnica de análisis discriminante. A continuación se presentan los referentes teóricos que en materia financiera soportan esta investigación.

Los indicadores financieros son el producto de establecer resultados numéricos basados en relacionar dos cifras o cuentas bien sea del Balance General y/o del Estado de Pérdidas y Ganancias. Los resultados así obtenidos por sí solos no tienen mayor significado; sólo cuando son relacionados unos con otros y son comparados con los de años anteriores o con los de empresas del mismo sector y a su vez el analista se preocupa por conocer a fondo la operación de la compañía, se pueden obtener resultados significativos y sacar conclusiones sobre la situación financiera real de la empresa, siendo el objeto de estudio de esta investigación las empresas del sector alimento de Barranquilla [3].

En concordancia con lo anterior y teniendo en cuenta que la liquidez, actividad, rentabilidad y endeudamiento son indicadores financieros que permiten evaluar el desempeño organizacional de las empresas en términos de resultados, que en esencia es el propósito de la evaluación del mejoramiento de los indicadores seleccionados, en este trabajo se identificaron y calcularon los siguientes indicadores financieros. 


\section{Indicadores de liquidez}

Estos indicadores surgen de la necesidad de medir la capacidad que tienen las empresas para cancelar sus obligaciones a corto plazo. Sirven para establecer la facilidad o dificultad que presenta una compañía para pagar sus pasivos corrientes con el producto de convertir a efectivo sus activos corrientes. Determinan qué pasaría si a la empresa se le exigiera el pago inmediato de todas sus obligaciones a menos de un año. En la Tabla 1 se presentan las ecuaciones (1), (2) y (3) que indican cómo calcular los indicadores de liquidez (razón corriente, prueba ácida y razón de liquidez).

Tabla 1. Indicadores de liquidez.

\begin{tabular}{|l|ll|}
\hline Indicador & Ecuación \\
\hline $\begin{array}{l}\text { Razón } \\
\text { corriente }\end{array}$ & $\frac{\text { Activo Corriente }}{\text { Pasivo Corriente }} \times 100$ & $(1)$ \\
\hline $\begin{array}{l}\text { Prueba } \\
\text { ácida }\end{array}$ & $\frac{\text { Activo Corriente }- \text { Inventarios }}{\text { Pasivo Corriente }} \times 100$ \\
\hline $\begin{array}{l}\text { Razón de } \\
\text { liquidez }\end{array}$ & $\frac{\text { Banco }+ \text { Caja }}{\text { Pasivo Corriente }}$ \\
\hline
\end{tabular}

\section{Indicadores de actividad}

Estos indicadores, llamados también indicadores de rotación, tratan de medir la eficiencia con la cual una empresa utiliza sus activos, según la velocidad de recuperación de los valores aplicados en ellos. Se pretende imprimirle un sentido dinámico al análisis de la aplicación de recursos, mediante la comparación entre cuentas de balance (estáticas) y cuentas de resultado (dinámicas), como se muestra en la Tabla 2, ecuación (4).

Tabla 2. Indicador de actividad.

\begin{tabular}{|l|l|}
\hline Indicador & Ecuación \\
\hline Rotación de activos & $\frac{\text { Ventas }}{\text { Total activos }}$ \\
\hline
\end{tabular}

\section{Indicadores de rentabilidad}

Los indicadores de rentabilidad, denominados también de rendimiento o lucratividad, sirven para medir la efectividad de la administración de la empresa para controlar el costo y el gasto, y de esta manera convertir las ventas en utilidades [4]; como se muestra en la Tabla 3, ecuación (5).
Tabla 3. Indicador de rentabilidad.

\begin{tabular}{|l|l|}
\hline Indicador & Ecuación \\
\hline $\begin{array}{l}\text { Rentabilidad } \\
\text { operativa del activo }\end{array}$ & $\frac{\text { Utilidad antes de impuesto }}{\text { Total activos }}$ \\
\hline
\end{tabular}

\section{Indicadores de endeudamiento}

Los indicadores de endeudamiento tienen por objeto medir en qué grado y de qué forma participan los acreedores dentro del financiamiento de la empresa. De la misma manera, se trata de establecer el riesgo que corren tales acreedores, el riesgo de los dueños y la conveniencia o inconveniencia de un determinado nivel de endeudamiento para la empresa, como se muestra en la Tabla 4, ecuación (6).

Tabla 4. Indicador de endeudamiento.

\begin{tabular}{|l|l|}
\hline Indicador & Ecuación \\
\hline $\begin{array}{l}\text { Nivel de } \\
\text { endeudamiento }\end{array}$ & $\frac{\text { Pasivo total }}{\text { Activo total }}$ \\
\hline
\end{tabular}

\section{Indicadores de apalancamiento}

Estos indicadores comparan el financiamiento originario de terceros con los recursos del accionista, socios o dueños de las empresas, con el fin de establecer cuál de las dos partes está corriendo el mayor riesgo. Así, si los accionistas contribuyen apenas con una pequeña parte del financiamiento total, los riesgos de la empresa recaen principalmente sobre los acreedores, como se muestra en la Tabla 5, ecuaciones (7) y (8).

Tabla 5. Indicadores de apalancamiento.

\begin{tabular}{|l|l|}
\hline \multicolumn{1}{|c|}{ Indicador } & Ecuación \\
\hline $\begin{array}{l}\text { Apalancamiento a } \\
\text { corto plazo }\end{array}$ & $\frac{\text { Pasivo corriente }}{\text { Total activo }}$ \\
\hline $\begin{array}{l}\text { Apalancamiento a } \\
\text { largo plazo }\end{array}$ & $\frac{\text { Pasivo no corriente }}{\text { Total activo }}$ \\
\hline
\end{tabular}

Criterios para la aplicación del análisis discriminante en la evaluación del mejoramiento de los indicadores de liquidez, actividad, rentabilidad y endeudamiento en las empresas del sector alimento de Barranquilla

Se puede definir la evaluación como un proceso orientado a medir los resultados generados (cambios y causas) por las acciones formativas, 
desarrolladas en el escenario socioprofesional de las organizaciones [5].

En este sentido, analizar el impacto de los indicadores financieros en los ingresos y crecimiento de las empresas es importante, porque sirve para adoptar y mejorar estándares o hacer ajustes en los sistemas organizacionales y específicamente en el mejoramiento de los indicadores de liquidez, actividad, rentabilidad y endeudamiento.

Evaluar el mejoramiento de los indicadores financieros en los ingresos y crecimiento de las empresas es importante con el fin de adoptar y mejorar estándares o hacer ajustes en los sistemas organizacionales y específicamente en las empresas del sector analizado, de tal forma que reflejen eficiencia, eficacia y calidad en donde se implementen.

\section{Análisis discriminante}

En esta investigación se utilizó el análisis discriminante, el cual es una técnica de análisis multivariante que procura encontrar relaciones lineales entre las variables continuas que mejor discriminen en los grupos categóricos previamente definidos. Siendo el análisis discriminante una técnica estadística, que fue introducida por R.A. Fisher en 1936 en el primer tratamiento moderno de problemas separatorios, cuya finalidad es analizar si existen diferencias significativas entre grupos de objetos respecto a un conjunto de variables medidas sobre los mismos y en el caso de que existan explicar en qué sentido se dan y proporcionar procedimientos de clasificación sistemática de nuevas observaciones de origen desconocido en uno de los grupos analizados. La variable dependiente de clasificación es una variable no métrica, mientras que las variables independientes se supone que son métricas. Para el caso de esta investigación se analiza el comportamiento en dos periodos distintos de los indicadores financieros seleccionados para las empresas del sector alimento de Barranquilla [6-7].

De igual forma, los objetivos primarios del análisis discriminante son la descripción de las diferencias entre grupos y la predicción de pertinencia a los grupos [8-9]. El otro objetivo consiste en determinar una o más ecuaciones matemáticas, funciones discriminantes, que permitan la clasificación de nuevos casos a partir de la información que se tiene de ellos, estableciendo la solvencia e insolvencia con la mayor precisión posible, modelo matemático que se presenta en las ecuaciones (10) y (11).

Asimismo, Pérez [10], Mateos, Iturrioz y Gimeneo [11] señalan que la variable dependiente de clasificación es una variable no métrica, mientras que las variables independientes se supone que son métricas y de esta manera se determina si existen diferencias significativas que permitan analizar el comportamiento del grupo de empresas estudiadas.

\section{Distancia de Mahalanobis}

Como criterio de selección de variables que mejor discriminan los indicadores de liquidez, actividad, rentabilidad y endeudamiento en la aplicación del análisis discriminante para evaluar el mejoramiento de los indicadores financieros de las empresas del sector alimento de Barranquilla, se utilizó la distancia $\mathrm{D}^{2}$ de Mahalanobis que es una medida de distancia generalizada y se basa en la distancia euclidiana al cuadrado que se adecúa a varianzas desiguales; la regla de selección en este procedimiento es maximizar la distancia $\mathrm{D}^{2}$ de Mahalanobis. La distancia multivariante entre los grupos $a$ y $b$ se define como, ecuación (9):

$$
\begin{aligned}
& D=(n-k) \sum_{i=1}^{p} \sum_{j=1}^{p} W_{i j}^{-1}\left(X_{i}^{(a)}-\right. \\
& \left.X_{i}^{(b)}\right)\left(X_{j}^{(a)}-X_{j}^{(b)}\right)
\end{aligned}
$$

Donde $n$ es el número de casos válidos, $k$ es el número de grupos, $X_{i}^{(a)}$ es la media del grupo $a$ en la i-ésima variable independiente, $X_{i}^{(b)}$ es la media del grupo $b$ en la i-ésima variable independiente, y $W_{i j}^{-1}$ es un elemento de la inversa de la matriz de varianzas-covarianzas intra-grupos [12]. Siendo la variabilidad total de la forma, ecuación (10).

$$
T_{i j}=W_{i j}+V_{i j}
$$

La covarianza total es igual a la covarianza dentro de grupos más la covarianza entre grupos.

Así, la probabilidad $P\left(K_{i} / D\right)$ de que un objeto $j$, con una puntuación discriminante $D=\left(y_{j 1}, \ldots, y_{j m}\right)$ pertenezca al grupo i-ésimo se puede estimar mediante la regla de Bayes:

$$
P\left(K_{i} / D\right)=\frac{P\left(D / K_{i}\right) P\left(K_{i}\right)}{\sum_{i}^{m} P\left(D / K_{i}\right) P\left(K_{i}\right)}
$$


$P\left(K_{i}\right)$ es la probabilidad a priori y es una estimación de la confianza de que un objeto pertenezca a un grupo si no se tiene información previa.

Como cualquier otra técnica estadística, la aplicación de la misma ha de ir precedida de una comprobación de los supuestos asumidos por el modelo. El análisis discriminante se apoya en los siguientes supuestos:

- Normalidad multivariante.

- Igualdad de matrices de varianza-covarianza.

- Linealidad.

- Ausencia de multicolinealidad y singularidad.

Finalmente, los indicadores financieros son de gran utilidad para la identificación de nuevas necesidades de medición y control, tal como lo argumenta Triana [13], quien explica que los indicadores son herramientas para el ciclo interminable de información al interior de la empresa "El desarrollo de estos indicadores son parte fundamental en el mejoramiento de la calidad, debido a que son medios económicos y rápidos de identificación de problemas, según la naturaleza y manejo del mismo". En este sentido la metodología que se propone constituye una herramienta valiosa en el análisis y mejoramiento de la gestión financiera de las organizaciones.

A continuación se estructura y propone el modelo para la aplicación del análisis discriminante en la evaluación del mejoramiento de los indicadores financieros en las empresas del sector alimento de Barranquilla, en los años 2004 y 2009, y de esta manera determinar si existen diferencias significativas que permitieron analizar el comportamiento del grupo de empresas estudiadas.

\section{METODOLOGÍA}

Esta es una investigación en donde se utiliza un análisis cualitativo, descriptivo propositivo y cuantitativo, soportada en un análisis discriminante multivariado (ADM). Para contrastar si en dos periodos distintos se puede evidenciar la evolución de los estados financieros como resultado de la evaluación del mejoramiento de los indicadores de liquidez, actividad, rentabilidad y endeudamiento en el sector analizado, para lo que se estudiaron los rubros requeridos de las empresas del sector alimento de Barranquilla [14].
Para la realización de esta investigación se utilizaron los indicadores presentados a continuación, utilizando el ADM, para hallar la estimación de los años evaluados, como se muestra en la Tabla 6 .

Tabla 6. Variable e indicadores utilizados para realizar el análisis de discriminante.

\begin{tabular}{|c|l|}
\hline Variable & \multicolumn{1}{|c|}{ Indicador } \\
\hline RC & Razón corriente \\
\hline PA & Prueba ácida \\
\hline RO & Rentabilidad operativa del activo \\
\hline RA & Rotación de activo \\
\hline NE & Nivel de endeudamiento \\
\hline ACP & Apalancamiento corto plazo \\
\hline ALP & Apalancamiento largo plazo \\
\hline
\end{tabular}

\section{Población}

La población de esta investigación está conformada por 13 empresas del sector alimento de Barranquilla que presentaron sus estados financieros entre el año 2004 y 2009.

\section{Fuentes y datos}

Asimismo, se tomaron como fuentes los estados financieros de la Superintendencia de Sociedades [15] y la Cámara de Comercio de las empresas seleccionadas, que proyectaron sus estados financieros del 2004 y 2009. De donde se tomaron los diferentes rubros de los estados financieros de las organizaciones objeto de estudio, labor que tomó cuatro meses de trabajo. Inicialmente se obtuvieron los rubros financieros de los estados financieros, para posteriormente calcular los indicadores seleccionados.

\section{Análisis}

Para analizar las variables asociadas a los indicadores seleccionados se utilizó el análisis discriminante por medio de software SPSS statistics 19, aplicación con la cual se establecieron las funciones discriminantes y se estudiaron los diferentes estadísticos; esta técnica permite la estimación en un marco único analizar si los indicadores financieros evaluados en el mismo contexto presentan diferencias significativas en los dos periodos seleccionados. Seguidamente se procedió a verificar el cumplimiento de los supuestos requeridos para aplicar el análisis discriminante y de esta forma calcular y establecer 
la función objetivo, con el fin de determinar qué indicadores discriminaban mejor para evaluar el mejoramiento de los indicadores financieros en las empresas del sector alimento de la ciudad de Barranquilla. También se calculó la precisión del modelo para predecir el comportamiento futuro de los indicadores en el sector. Por último, con los estadísticos, específicamente las medias de los indicadores para el sector, se analizó y evaluó el comportamiento de los diferentes indicadores financieros en los dos periodos 2004-2009.

\section{RESULTADOS}

Aplicación del análisis discriminante para evaluar el mejoramiento de los indicadores financieros en las empresas del sector Alimento de Barranquilla

\section{Verificación de los supuestos}

En las Tablas 7 y 8 se presentan los resultados de normalidad para los datos de los periodos 2004 y 2009 respectivamente, asociados a la prueba de Shapiro \& Wilk que requiere que los estadísticos estén por encima de 0,8 . Las pruebas de normalidad para las variables por separado de los dos periodos respectivos dan como resultado que algunas no se comportan como variables normales, por lo que se viola el supuesto de multinormalidad. Sin embargo, P. Lachenbruch [16] ha demostrado que el análisis discriminante no es particularmente sensible a las violaciones de menor importancia de la hipótesis de normalidad. B. Tabachnick \& L. Fidell [17] hacen algunas precisiones acerca de la robustez de esta técnica en relación con el tamaño de las muestras, sugieren un tamaño de muestra pequeño de grupos similares para que el modelo sea robusto ante la violación del supuesto de multinormalidad.

Tabla 7. Prueba de Shapiro \& Wilk para la comprobación de la normalidad de las razones financieras del año 2004.

\begin{tabular}{|c|c|c|c|}
\hline \multirow{2}{*}{} & \multicolumn{3}{|c|}{ Shapiro-Wilk } \\
\cline { 2 - 4 } & Estadístico & gl & Sig. \\
\hline RC & 0,893 & 13 & 0,106 \\
\hline PA & 0,811 & 13 & 0,009 \\
\hline RO & 0,693 & 13 & 0 \\
\hline RA & 0,937 & 13 & 0,417 \\
\hline NE & 0,805 & 13 & 0,008 \\
\hline ACP & 0,743 & 13 & 0,002 \\
\hline ALP & 0,545 & 13 & 0 \\
\hline
\end{tabular}

Tabla 8. Prueba de Shapiro \& Wilk para la comprobación de la normalidad de las razones financieras del año 2009.

\begin{tabular}{|c|c|c|c|}
\hline \multirow{2}{*}{} & \multicolumn{3}{|c|}{ Shapiro-Wilk } \\
\cline { 2 - 4 } & Estadístico & gl & Sig. \\
\hline RC & 0,929 & 13 & 0,329 \\
\hline PA & 0,943 & 13 & 0,497 \\
\hline RO & 0,96 & 13 & 0,761 \\
\hline RA & 0,911 & 13 & 0,188 \\
\hline R5 & 0,943 & 13 & 0,503 \\
\hline ACP & 0,842 & 13 & 0,023 \\
\hline ALP & 0,691 & 13 & 0 \\
\hline
\end{tabular}

\section{Homogeneidad de matrices de varianza-covarianza}

El supuesto de igualdad de matrices de varianzacovarianza para los dos años 2004 y 2009 se comprobó con la prueba de Box, como se muestra en la Tabla 9.

Tabla 9. Resultados de la prueba de Box.

\begin{tabular}{|c|c|c|}
\hline \multicolumn{2}{|c|}{ M de Box } & 136,287 \\
\hline \multirow{4}{*}{ F } & Aprox. & 3,006 \\
\cline { 2 - 3 } & gl1 & 28 \\
\cline { 2 - 3 } & gl2 & 1308,838 \\
\cline { 2 - 3 } & Sig. &, 000 \\
\hline
\end{tabular}

Los resultados obtenidos del estadístico de contraste $\mathrm{M}=136,287$ y un valor de $\mathrm{F}=3,006$ con una probabilidad asociada $\mathrm{p}=0,00$ permiten aceptar con un alto grado de confianza la hipótesis nula de que no existen diferencias entre las matrices de covarianza de los dos grupos.

\section{Linealidad y multicolinealidad y singularidad}

Los supuestos de linealidad y multicolinealidad y singularidad no serán revisados, dado que para la construcción del modelo de análisis discriminante se utilizará el método por etapas o paso a paso, teniendo en cuenta el criterio de tolerancia para seleccionar las variables que son incluidas. De este modo, aquellas variables que presentan una correlación múltiple elevada con las restantes variables arrojarán una baja tolerancia y no serían consideradas de cara a la construcción de la función discriminante [18].

Selección de las variables que mejor discriminan Para determinar qué variables discriminan independientemente entre los grupos de "2004" y "2009" se estima la distancia $\mathrm{D}^{2}$ de Mahalanobis y el Lambda Wilks para cada una de ellas, su 
correspondiente razón $\mathrm{F}$ y nivel de significancia para rechazar la hipótesis nula de que las observaciones provienen de la misma población. Se va a considerar un nivel de significación de 0,05 para los estadísticos F para entrar y F para eliminar. Como se mencionó anteriormente el criterio de selección de las variables es el de la distancia $\mathrm{D}^{2}$ de Mahalanobis; se utilizaron todas las variables seleccionadas (Razón Corriente (RC), Prueba Acida (PA), Rentabilidad Operativa del Activo (RO), Rotación de Activo (RA), Nivel de Endeudamiento (NE), Apalancamiento Corto Plazo (ACP), Apalancamiento Largo Plazo (APL)), siendo las variables que mejor discriminan la Rentabilidad Operativa del Activo (RO), Rotación de Activo (RA), Nivel de Endeudamiento (NE), constituyendo éstas las funciones discriminantes; el resultado final del modelo se muestra en la Tabla $10 \mathrm{y}$ en las ecuaciones (12) y (13).

Tabla 10. Coeficientes de la función de clasificación.

\begin{tabular}{|c|c|c|}
\hline \multirow{2}{*}{} & \multicolumn{2}{|c|}{ Año } \\
\cline { 2 - 3 } & $\mathbf{2 0 0 4}$ & \multicolumn{1}{c|}{$\mathbf{2 0 0 9}$} \\
\hline RC & 34,581 & 28,459 \\
\hline PA & $-5,333$ & $-5,483$ \\
\hline RO & 3,181 & 4,485 \\
\hline RA & $-3,167$ & $-2,913$ \\
\hline NE & $-20,708$ & $-15,585$ \\
\hline ACP & 170,202 & 157,438 \\
\hline ALP & 68,350 & 64,704 \\
\hline (Constante) & $-87,405$ & $-75,060$ \\
\hline
\end{tabular}

Con base en los resultados anteriores se procedió a calcular las funciones discriminantes para evaluar el mejoramiento de los indicadores financieros en las empresas del sector alimento de Barranquilla, ecuaciones (12) y (13).

$\mathrm{Z}_{1}=\mathrm{RC} *(34,581)+\mathrm{PA} *(-5,333)+\mathrm{RO} *(3,181)$

$+\mathrm{RA}^{*}(-3,167)+\mathrm{NE} *(-20,708)+\mathrm{ACP} *(170,202)$ $+\operatorname{ALP}^{*}(68,350)$

$\mathrm{Z}_{2}=\mathrm{RC} *(28,459)+\mathrm{PA} *(-5,483)+\mathrm{RO} *(4,485)$

$+\mathrm{RA}^{*}(-2,913)+\mathrm{NE} *(-15,585)+\mathrm{ACP} *(157,438)$

+ ALP $*(64,704)$

En el Anexo 1 y Anexo 2 se muestran los indicadores financieros en las empresas del sector alimentos de Barranquilla.
De este modo, la capacidad de clasificación del modelo fue excelente, generando como resultado un error Tipo I de 100,0 y un error Tipo II de 70,0\% para una efectividad de clasificación de $85,0 \%$, como se muestra en la Tabla 11.

Tabla 11. Resultados de la clasificación.

\begin{tabular}{|c|c|c|c|c|c|}
\hline & & \multirow[t]{2}{*}{ Año } & \multicolumn{2}{|c|}{$\begin{array}{l}\text { Grupo de pertenencia } \\
\text { pronosticado }\end{array}$} & \multirow[t]{2}{*}{ Total } \\
\hline & & & 2004 & 2009 & \\
\hline \multirow{6}{*}{ 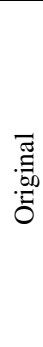 } & \multirow{3}{*}{$\begin{array}{l}\stackrel{0}{*} \\
\stackrel{0}{0} \\
\stackrel{0}{\mathscr{U}} \\
\end{array}$} & 2004 & 13 & 0 & 13 \\
\hline & & 2009 & 3 & 7 & 10 \\
\hline & & $\begin{array}{c}\text { Casos } \\
\text { desagrupados }\end{array}$ & 1 & 2 & 3 \\
\hline & \multirow{3}{*}{$\%$} & 2004 & 100,0 &, 0 & 100,0 \\
\hline & & 2009 & 30,0 & 70,0 & 100,0 \\
\hline & & $\begin{array}{c}\text { Casos } \\
\text { desagrupados }\end{array}$ & 33,3 & 66,7 & 100,0 \\
\hline
\end{tabular}

Evaluación de los indicadores seleccionados en las empresas del sector alimento de Barranquilla Cuando se revisan los estadísticos (Media) de los indicadores seleccionados de las empresas en Barranquilla, se observa que existe un mejoramiento en el indicador Apalancamiento a Largo Plazo (ALP), lo que no ocurre para los indicadores de Razón Corriente (RC), Prueba Acida (PA), Rentabilidad Operativa del Activo (RO), Rotación de Activo (RA), Nivel de Endeudamiento (NE), Apalancamiento Corto Plazo (ACP) como se muestra en la Tabla 12.

Tabla 12. Medias de los indicadores financieros.

\begin{tabular}{|c|l|r|r|}
\hline \multicolumn{2}{|c|}{ Año } & \multicolumn{1}{c|}{ Media } & Desv. típ. \\
\hline \multirow{7}{*}{2004} & RC & 1,5690 &, 77126 \\
\cline { 2 - 4 } & PA & 1,1780 &, 83613 \\
\cline { 2 - 4 } & RO &, 0581 &, 25756 \\
\cline { 2 - 4 } & RA & 2,7156 & 1,21469 \\
\cline { 2 - 4 } & NE & 1,4274 &, 78527 \\
\cline { 2 - 4 } & ACP &, 8995 &, 14596 \\
\cline { 2 - 4 } & ALP &, 1510 &, 32054 \\
\hline \multirow{7}{*}{2009} & RC & 1,1170 &, 24043 \\
\cline { 2 - 4 } & PA &, 8265 &, 25223 \\
\cline { 2 - 4 } & RO &, 0370 &, 12161 \\
\cline { 2 - 4 } & RA & 2,6550 & 1,62561 \\
\cline { 2 - 4 } & NE & 1,3508 &, 30429 \\
\cline { 2 - 4 } & ACP &, 8156 &, 19867 \\
\cline { 2 - 4 } & ALP &, 3353 &, 55389 \\
\hline
\end{tabular}




\section{DISCUSIÓN}

$\mathrm{Al}$ analizar las funciones discriminantes generadas en la Tabla 10 se puede concluir que indicadores como la Rentabilidad Operativa del Activo (RO), Rotación de Activo (RA), Nivel de Endeudamiento (NE) discriminan bien mejorando de un periodo a otro. Sin embargo, para el caso de la Razón Corriente (RC), Prueba Acida (PA), Apalancamiento Corto Plazo (ACP), Apalancamiento Largo Plazo (APL) se puede observar que estos indicadores no evidencian el mismo comportamiento en el transcurso de los años 2004 y 2009.

En lo que respecta a las medias presentadas en la Tabla 12 de los indicadores seleccionados se pudo analizar que el indicador Apalancamiento Largo Plazo (ALP) mejoró (aunque de manera mínima) de un periodo a otro, por lo que se infiere que existe un mejoramiento en el sector para este indicador. Sin embargo, en los otros indicadores no se observa un mejoramiento en el periodo.

Particularmente, en el indicador Razón Corriente (RC) se observa una disminución de 1,569 a 1,117 en los dos periodos analizados, mostrando un deterioro en la liquidez y por tanto en la capacidad que tienen las empresas para cumplir con sus obligaciones financieras, deudas o pasivos a corto plazo. Estos resultados concuerdan con los del indicador Prueba Acida, en el que comparativamente se observa una disminución mayor de 1,178 a 0,8265. Asimismo, el Indicador Rentabilidad Operativa (RO) observa una disminución de 0,0581 a 0,37 resultados que muestran un deterioro en la rentabilidad de las empresas del sector analizado, lo cual puede estar relacionado con falta de controles efectivos para el manejo de los costos y los gastos de manera de convertir las ventas en utilidades. Adicionalmente el indicador Rotación de Activo (RA) muestra una disminución en los dos periodos analizados; esta situación puede estar asociada a pérdidas de mercado por parte de las empresas del sector alimentos de la ciudad de Barranquilla, representadas en una disminución de las ventas o en un aumento de las inversiones en activos fijos. Adicionalmente, el Nivel de Endeudamiento muestra un mejoramiento al disminuir en los periodos analizados, lo cual se relaciona con el planteamiento de aumento en las inversiones en activos realizado en el análisis del indicador de Rotación de Activo. Por su parte, el
Indicador de Apalancamiento a Corto Plazo (APC) y el Apalancamiento de Largo Plazo (APL) muestran un comportamiento inverso, disminuyendo para el caso de APC y aumentando para el APL; estos resultados muestran un aumento del pasivo corriente y una reducción del pasivo no corriente.

Es importante señalar que estudios similares de T. Fontalvo, A. Mendoza y J. Morelos [19] han demostrado que el indicador Razón Corriente es sensible y muestra mejoras significativas como efecto de la gestión en el sector estudiado, lo que no ocurre para esta investigación. Asimismo, T. Fontalvo, J. Morelos y E. De la Hoz [20] establecen que la aplicación del análisis discriminante en el mejoramiento de los indicadores financieros de las empresas permite identificar aquéllos con mayor impacto para la continuidad de las mismas en el mercado; por lo que es importante poder contar con herramientas que puedan pronosticar esta situación como las funciones discriminantes calculadas en esta investigación.

\section{CONCLUSIÓN}

En el análisis discriminante desarrollado en esta investigación para la construcción del modelo se utilizaron 13 empresas del sector alimento de Barranquilla; el modelo presentó una buena efectividad en la clasificación. En la muestra original la precisión del modelo en el 2004 es 100,0\% y en el 2009 es del 70,0\%, para un promedio total de clasificación del 85,0\% de las empresas, lo que demuestra la confiabilidad para predecir el comportamiento de los indicadores financieros en el sector a futuro.

De la aplicación del análisis discriminante para evaluar el mejoramiento de los indicadores financieros en las empresas de sector alimento de Barranquilla, se puede concluir que los indicadores financieros Rentabilidad Operativa del Activo (RO), Rotación de Activo (RA), Nivel de Endeudamiento (NE) del sector presentan diferencias significativas de un periodo a otro. Aunque solo la media del indicador financiero Apalancamiento a Largo Plazo (ALP) mejora de un periodo a otro en el sector estudiado.

Este trabajo se constituye en un referente para desarrollar otras investigaciones que evalúen no solo variables e indicadores financieros, sino 
otras variables internas y externas en diferentes sectores que permitan medir eficiencia, eficacia, productividad y competitividad. De igual forma, la investigación permitió establecer una función objetivo para las empresas evaluadas en el sector alimento de Barranquilla, con lo que se puede estudiar y analizar qué indicadores discriminan mejor y así poder tomar acciones teniendo en cuenta el cálculo y estudio de los indicadores seleccionados a futuro.

A partir de estudios como éste, se podrán realizar análisis en diferentes sectores empresariales que faciliten la toma de decisiones sobre qué variables, rubros e indicadores redundan en el mejoramiento de la situación financiera de las organizaciones y analizar otros indicadores como productividad, competitividad que incidan en el posicionamiento de otros sectores estudiados.

Se recomienda para futuros estudios incrementar el número de indicadores financieros e incorporar indicadores de productividad y competitividad; y se invita a analizar el comportamiento de otros sectores empresariales.

\section{REFERENCIAS}

[1] A. Martínez y E. Mario. "Ideas para el cambio y el aprendizaje en la organización, una perspectiva sistémica". Ed. ECOE Universidad de la Sabana. 1 Edición, p. 58. Bogotá, D.C. Colombia. 2002. ISBN: 958-8384-3.

[2] J. Fernández. "Liderazgo empresarial en tiempos de cambio según Sun Tsu". Harvard Deusto Business Review. Vol. 4, Issue 119, p. 104. August, 2008. ISSN: 0210-900X.

[3] X.Vásquez, A. Guerra y I. Ahmed. "Aplicación de métodos multivariados: una respuesta a las limitaciones de los ratios financieros". Revista de la Universidad de Granma. Vol. $24 \mathrm{~N}^{\circ} 114$, p. 44. Octubre 2008. ISSN: 1696-8360.

[4] H. Ortiz. "Análisis financiero aplicado y principios de administración financiera". Ed. Universidad Externado de Colombia. $\mathrm{N}^{\circ}$ Edición 14, p. 118. Bogotá, Colombia. 2011. ISBN: 978-958-710-650-3.

[5] J. Tejada y E. Ferrández. "La evaluación del impacto de la formación como estrategia de mejora en las organizaciones". Revista Electrónica de Investigación Educativa. Vol. 4
$\mathrm{N}^{\circ}$ 104, p. 20. Agosto 2007. URL: http:// redie.uabc.mx/vol9no2/contenido-tejada2. ht $\mathrm{ml}$. Fecha de consulta: 6 de agosto de 2010

[6] J. Mylonakis and G. Diacogiannis. "Evaluating the likelihood of using linear discriminant analysis as a commercial bank card owners credit scoring model". International business research. Vol. 3, Issue 2, p. 490. April, 2010. ISSN: 0047-2506.

[7] J. Suárez. "Los parámetros característicos de las empresas manufactureras de alta rentabilidad. Una aplicación del análisis discriminante". Revista Española de financiación y contabilidad. Vol. XXIX $\mathrm{N}^{\circ} 104$, pp. 443-481. Mayo de 2000. ISSN: 0210-2412.

[8] R. Mileris. "Estimation of loan applicants default probability applying discriminant analysis and simple Bayesian classifier". Economics and management. Vol. 33, Issue 302, p. 28. August, 2010. ISSN: 1822-6515.

[9] C. Peretto. "Utilización del análisis discriminante logístico para explorar las causas de la eficiencia del sistema bancario argentino". Cuaderno de Cimbage. Vol. $1 \mathrm{~N}^{\circ} 11$, pp. 39-57. Mayo de 2009. ISSN: $1669-1830$.

[10] C. Pérez. "Técnicas de análisis de datos con SPSS 15". Editorial Pearson, Prentice Hall. Edition No 5, p. 314. Spain. 2009. ISBN: 978-848-322-601-8.

[11] R. Mateos, J. Iturrioz y R. Gimeneo. "La participación financiera y el papel de la mujer en la toma de decisiones de las sociedades cooperativas: los consejos de administración". Revista Europea de dirección y economía de la empresa. Vol. $18 \mathrm{~N}^{\mathrm{o}} 3$, pp. 65-82. Noviembre de 2009. ISSN: 1019-6838.

[12] M. Tricova and D Terdovski. "Applie discriminant Analysis in estimation of potencial EU Members". Busines statisticsEconomic Informatics. Vol. 32, Issue 120, p. 48. June, 2008. ISSN: 1583-9982.

[13] L. Triana. "Valoración de Empresas mediante el análisis de Flujos de Caja. Preparación y Evaluación de Proyectos de Inversión: Casos Prácticos". Editorial Uniautónoma, p. 112. Barranquilla, Colombia. 2010. ISBN: 24978-958-8524-23-8. 
[14] B. Vuran. "Prediction of business failure: A comparison of discriminant and logistic regression analyses". Istanbul University journal of the school of business administration. Vol. 38, Issue 1, pp. 47-65. September, 2009. ISSN: 1303-1732.

[15] Superintendencia de Sociedades. URL: http:// sirem.supersociedades.gov.co/SIREM/. Fecha de consulta: 1 de octubre de 2008 .

[16] P. Lachenbruch. "Discriminant Analysis". Editorial Macmillan Pub Co., p. 47. New York, EE.UU. 1975. ISBN: 10: 0028482506.

[17] B. Tabachnick and L. Fidell. "Using Multivariate Statistics". Edition $\mathrm{N}^{\mathrm{o}}$ 4. Boston, EE.UU., p. 104. 2001. ISBN: 0-321-05677-9.

[18] J. Rodríguez y A. Moreno. "Fragilidad financiera de las firmas en Colombia, 20002006. Un análisis discriminante de un modelo
Minskano". Ed. Universidad Nacional de Colombia. Vol. $14 \mathrm{~N}^{\circ}$ 2, pp. 14-32. Marzo de 2011. ISSN: 0124-5821.

[19] T. Fontalvo, A. Mendoza y J. Morelos. "Evaluación del impacto de los sistemas de gestión de la calidad en la liquidez y rentabilidad de las empresas de la Zona Industrial de Mamonal (Cartagena-Colombia)". Revista Católica del Norte, Vol. $1 \mathrm{~N}^{\mathrm{o}}$ 34, pp. 1-28. Septiembre de 2011. ISSN: 0124-5821.

[20] T. Fontalvo, J. Morelos y E. De la Hoz. "Aplicación del análisis discriminante para evaluar el mejoramiento de los indicadores financieros en las empresas del sector extracción de petróleo crudo y gas natural en Colombia”. Revista Soluciones de Postgrado EIA. Vol. $1 \mathrm{~N}^{\mathrm{o}}$ 7, pp. 1-16. Julio de 2011. ISSN: 2811-3854

\section{ANEXOS}

Anexo 1. Indicadores Financieros del año 2004.

\begin{tabular}{|c|c|c|c|c|c|c|c|c|}
\hline & & $\begin{array}{c}\text { RAZÓN } \\
\text { CORRIENTE }\end{array}$ & $\begin{array}{l}\text { PRUEBA } \\
\text { ÁCIDA }\end{array}$ & \begin{tabular}{|c|} 
RENT. \\
OPERATTVA \\
DEL ACTIVO \\
\end{tabular} & $\begin{array}{c}\text { ROTACIÓN } \\
\text { DE ACTIVOS }\end{array}$ & $\begin{array}{c}\text { NIVEL DE } \\
\text { ENDEUDAMIENTO }\end{array}$ & $\begin{array}{l}\text { APALANCAMIENTO } \\
\text { CORTO PLAZO }\end{array}$ & $\begin{array}{l}\text { APALANCAMIENTO } \\
\text { LARGO PLAZO }\end{array}$ \\
\hline $\mathbf{N}^{\circ}$ & RAZÓN SOCIAL & $\mathbf{R C}$ & PA & RO & RA & NE & ACP & ALP \\
\hline 1 & $\begin{array}{l}\text { ATUNES Y } \\
\text { ENLATADOS DEL } \\
\text { CARIBE S.A. }\end{array}$ & 1,3261251 & 0,4631237 & 0,0838312 & 1,7965027 & 1,0836839 & 0,8171807 & 0,1687017 \\
\hline 2 & $\begin{array}{l}\text { CAFÉ UNIVERSAL } \\
\text { S. A. }\end{array}$ & 0,9339094 & 0,7914839 & $-0,022993$ & 2,3093422 & 0,9267435 & 0,9923269 & 0,0082796 \\
\hline 3 & $\begin{array}{l}\text { COMPAĐIA } \\
\text { ENVASADORA DEL } \\
\text { ATLANTICO LTDA }\end{array}$ & 1,2234322 & 0,8430586 & 0,0385488 & 0,7585933 & 1,2234322 & 1 & 0 \\
\hline 4 & $\begin{array}{l}\text { FRIGORIFICOS DE } \\
\text { LA COSTA S. A. }\end{array}$ & 1,2352771 & 0,8404511 & 0,0189649 & 1,824696 & 1,214478 & 0,9831624 & 0,0138641 \\
\hline 5 & GELCO S.A. & 2,6959123 & 2,0274563 & 0,2390649 & 2,5656793 & 2,6959123 & 1 & 0 \\
\hline 6 & $\begin{array}{l}\text { GENEROSO } \\
\text { MANCINI \& CIA } \\
\text { LTDA }\end{array}$ & 2,046469 & 1,7709428 & 0,1516773 & 3,8477892 & 1,7708165 & 0,8653034 & 0,0760647 \\
\hline 7 & $\begin{array}{l}\text { GRASAS Y ACEITES } \\
\text { VEGETALES S.A. }\end{array}$ & 1,3387328 & 0,9922857 & 0,1864762 & 3,3827077 & 1,3068801 & 0,9762068 & 0,0182061 \\
\hline 8 & $\begin{array}{l}\text { GRUPO } \\
\text { ALIMENTARIO DEL } \\
\text { ATLANTICO S A }\end{array}$ & 1,2924906 & 0,72917 & 0,0161157 & 2,0539663 & 1,2924906 & 1 & 0 \\
\hline 9 & $\begin{array}{l}\text { LACTEOS DEL } \\
\text { CAMPO S.A. }\end{array}$ & 0,4438562 & 0,350073 & $-0,729315$ & 5,641962 & 0,2939124 & 0,6621794 & 1,1493922 \\
\hline 10 & $\begin{array}{l}\text { MOLINOS } \\
\text { BARRANQUILLITA } \\
\text { S.A. }\end{array}$ & 1,4431082 & 0,7804886 & 0,0760195 & 3,0958168 & 1,2092939 & 0,8379787 & 0,1339801 \\
\hline 11 & $\begin{array}{l}\text { MOLINOS DEL } \\
\text { ATLANTICO S. A. }\end{array}$ & 1,0859038 & 0,6051607 & 0,2822008 & 3,2535641 & 1,0859038 & 1 & 0 \\
\hline 12 & $\begin{array}{l}\text { OLEOFLORES } \\
\text { LIMITADA }\end{array}$ & 1,9972977 & 1,7855585 & 0,1302778 & 2,9647901 & 1,1174583 & 0,5594851 & 0,3942115 \\
\hline 13 & $\begin{array}{l}\text { PANIFICADORA } \\
\text { DEL LITORAL S.A. }\end{array}$ & 3,3350772 & 3,3350772 & 0,28481 & 1,8070819 & 3,3350772 & 1 & 0 \\
\hline
\end{tabular}


Anexo 2. Indicadores Financiero del año 2009.

\begin{tabular}{|c|c|c|c|c|c|c|c|c|}
\hline & & \begin{tabular}{|c|} 
RAZÓN \\
CORRIENTE
\end{tabular} & $\begin{array}{c}\text { PRUEBA } \\
\text { ÁCIDA }\end{array}$ & $\begin{array}{c}\text { RENT. } \\
\text { OPERATIVA } \\
\text { DEL ACTIVO }\end{array}$ & $\begin{array}{c}\text { ROTACIÓN } \\
\text { DE ACTIVOS }\end{array}$ & $\begin{array}{l}\text { NIVEL DE } \\
\text { ENDEUDA- } \\
\text { MIENTO }\end{array}$ & $\begin{array}{l}\text { APALANCAMIENTO } \\
\text { CORTO PLAZO }\end{array}$ & $\begin{array}{l}\text { APALANCAMIENTO } \\
\text { LARGO PLAZO }\end{array}$ \\
\hline $\mathbf{N}^{\circ}$ & RAZÓN SOCIAL & RC & PA & RO & $\mathbf{R A}$ & NE & ACP & ALP \\
\hline 1 & $\begin{array}{c}\text { ATUNES Y } \\
\text { ENLATADOS DEL } \\
\text { CARIBE S.A. }\end{array}$ & 1,2208142 & 0,5070925 & $-0,192349$ & 1,048303 & 1,1131346 & 0,807996 & 0,1946487 \\
\hline 2 & $\begin{array}{c}\text { CAFÉ UNIVERSAL } \\
\text { S. A. }\end{array}$ & 0,8848017 & 0,81312 & $-0,082063$ & 1,0365384 & 1,8824384 & 0,672175 & 0,5512057 \\
\hline 3 & $\begin{array}{c}\text { COMPAĐIA } \\
\text { ENVASADORA DEL } \\
\text { ATLANTICO LTDA }\end{array}$ & 1,1140025 & 0,9077545 & 0,2658318 & 1,3818439 & 1,6792944 & 1 & 0 \\
\hline 4 & $\begin{array}{l}\text { FRIGORIFICOS DE } \\
\text { LA COSTA S. A. }\end{array}$ & 1,3753141 & 1,0215321 & 0,0241618 & 1,9500909 & 1,2425317 & 0,9268809 & 0,0573595 \\
\hline 5 & GELCO S.A. & 0,8499985 & 0,7191352 & 0,1136437 & 2,0841651 & 1,6105239 & 0,8635562 & 0,1858853 \\
\hline 6 & $\begin{array}{l}\text { GENEROSO } \\
\text { MANCINI \& CIA } \\
\text { LTDA }\end{array}$ & 1,5596197 & 1,4188655 & 0,0430212 & 2,9266162 & 1,4918155 & 0,5942964 & 0,4377106 \\
\hline 7 & $\begin{array}{l}\text { GRASAS Y ACEITES } \\
\text { VEGETALES S.A. }\end{array}$ & 0,9997892 & 0,7734933 & 0,0942478 & 3,8941772 & 1,1433383 & 0,9842512 & 0,0160042 \\
\hline 8 & $\begin{array}{c}\text { GRUPO } \\
\text { ALIMENTARIO DEL } \\
\text { ATLANTICO S A }\end{array}$ & 1,157638 & 0,7006149 & 0,0097458 & 2,7034718 & 1,1039841 & 1 & 0 \\
\hline 9 & $\begin{array}{l}\text { LACTEOS DEL } \\
\text { CAMPO S.A. }\end{array}$ & 0,8030294 & 0,6194182 & 0,003782 & 6,4424119 & 0,924092 & 0,4065017 & 1,8181329 \\
\hline 10 & $\begin{array}{c}\text { MOLINOS } \\
\text { BARRANQUILLITA } \\
\text { S.A. }\end{array}$ & 1,2045234 & 0,7844451 & 0,0894925 & 3,0824648 & 1,3165893 & 0,9000953 & 0,0921472 \\
\hline 11 & $\begin{array}{l}\text { MOLINOS DEL } \\
\text { ATLANTICO S. A. }\end{array}$ & 1,6630614 & 1,1680662 & 0,1975438 & 3,8965578 & 1,4874041 & 1 & 0 \\
\hline 12 & $\begin{array}{l}\text { OLEOFLORES } \\
\text { LIMITADA }\end{array}$ & 0,848674 & 0,8064973 & 0,239869 & 5,4607009 & 2,1331744 & 0,5524642 & 0,9545149 \\
\hline 13 & $\begin{array}{c}\text { PANIFICADORA } \\
\text { DEL LITORAL S.A. }\end{array}$ & 1,1408056 & 1,1408056 & 0,2134518 & 1,6325805 & 2,2793478 & 1 & 0 \\
\hline
\end{tabular}

